\title{
Subendocardial Systolic Hypoperfusion in Stenotic Coronary Territories: Demonstration by 256-Slice Coronary CT Angiography
}

Yuzo Yamasaki ${ }^{1}$, Michinobu Nagao ${ }^{2 *}$, Yoshio Matsuo ${ }^{1}$, Takeshi Kamitani ${ }^{1}$, Masato Yonezawa ${ }^{1}$, Hiroshi Honda ${ }^{1}$, Satoshi Kawanami², Tetsuya Matoba ${ }^{3}$, Hidetake Yabuuchi ${ }^{4}$, Mani Vembar ${ }^{5}$ and Ko Higuchi ${ }^{5}$

${ }^{1}$ Department of Clinical Radiology, Graduate School of Medical Sciences, Kyushu University, Japan

${ }^{2}$ Molecular Imaging \& Diagnosis, Graduate School of Medical Sciences, Kyushu University, Japan

${ }^{3}$ Department of Cardiovascular Medicine, Graduate School of Medical Sciences, Kyushu University, Japan

${ }^{4}$ Department of Health science, Graduate School of Medical Sciences, Kyushu University, Japan

${ }^{5}$ Department of CT Clinical Science, Philips Healthcare, Cleveland, Ohio, USA

Received: November 18, 2013; Accepted: June 24, 2014, Published: July 2, 2014

*Corresponding author: Michinobu Nagao, Department of Molecular Imaging \& Diagnosis, Graduate School of Medical Sciences, Kyushu University, 3-1-1 Maidashi, Higashi-Ku Fukuoka-city, Fukuoka, 812-8582, Japan, Tel: +(81)-92-642-5695; Fax: (81)-92-642-5706; E-mail: minagao@radiol.med.kyushu-u. ac.jp

\begin{abstract}
Purpose: We propose a new, Myocardial Perfusion Imaging (MPI) with coronary CTA, and investigate the diagnostic ability of subendocardial hypoperfusion for Coronary Artery Disease (CAD) based on degree of coronary stenosis.

Methods: In twenty CAD patients, Coronary CTA and Invasive Coronary Angiography (CAG) were performed. CT-Myocardial Perfusion Image (MPI) was reconstructed at 4 cardiac phases $(0 \%$, $40 \%, 60 \%$, and $80 \%$ of the R-R interval), applying CT number measured in Hounsfield Unit (HU) as an estimate of perfusion. A myocardial segment with subendocardial low attenuation at endsystole was defined as subendocardial-systolic-hypoperfusion (SSH).

Results: Using $>50 \%$ stenosis on CAG as standard, SSH diagnosed stenotic coronary territories with $63 \%$ sensitivity, $82 \%$ specificity, and $70 \%$ accuracy. Using $>75 \%$ stenosis on CAG as standard, SSH diagnosed stenotic coronary territories with $73 \%$ sensitivity, $80 \%$ specificity, and $77 \%$ accuracy. The use of the SSH sign led to accurate diagnosis (result of CAG) in 9 of 60 territories (15\%), which could not be assessed due to severe coronary calcification.
\end{abstract}

Conclusion: SSH is frequently seen in the stenotic coronary territories. SSH might provide powerful information when the assessment of coronary artery is made difficult by severe calcification.

Keywords: Coronary CT; Myocardial perfusion; Myocardial ischemia, Invasive coronary angiography

\section{Introduction}

While Multi-slice Computed Tomography (MSCT) is increasingly being used as a noninvasive alternative to coronary angiography, several recent studies have shown the potential of MSCT to provide myocardial perfusion information from the same scan, which can be used in the diagnosis and evaluation of
CAD [1]. Coronary CT angiography CCTA is widely used as noninvasive imaging for assessment of coronary arteries. Newer MSCT scanners (64-slice and greater) with a high temporal resolution have improved the reliability of CCTA. However, CCTA still has several limitations. Motion artifacts, arrhythmia, and severe calcified plaque sometimes make assessment of coronary artery difficult. A recent study reported that a pattern of subendocardial hypoperfusion at systole in rest CCTA may characterize ischemic myocardium detected by SPECT [2,3]. But we hypothesize that SSH is observed in coronary stenotic territories because SSH probably represents reduction of myocardial blood flow to the myocardium. Furthermore, SSH has not yet been compared with the result of CAG and presence of SSH on CT is assessed visually, not by a quantitative method. The objective of this study was to quantify SSH related to stenotic coronary territories using retrospectively ECG-gated 256-slice CCTA and investigate its diagnostic capability for detecting Coronary Artery Disease (CAD) using CAG as a reference standard.

\section{Materials and Methods}

\section{Patient population}

Twenty consecutive patients with suspected CAD who underwent both CCTA and CAG within 3 months in our hospital between March 2010 and March 2012 were enrolled in this retrospective study. In all patients, Percutaneous Coronary Intervention or Coronary Artery Bypass Grafting (CABG) were not performed between the two examinations. Patients who had a history of prior Myocardial Infarction (MI) or had undergone CABG or stent placement were excluded. After approval from the local ethics committee, informed consent was obtained from all patients. Motion artifact and streak artifact was visually assessed, 
and cases with obvious artifact were excluded from our study The patient characteristics are shown in Table 1.

\section{CCTA Acquisition Protocol}

All CCTA examinations were performed using a 256-slice MSCT scanner (Brilliance iCT: Philips Healthcare, Cleveland, OH, USA). To prepare for the scan, all patients were administered sublingual nitroglycerin (5 mg) unless their systolic blood pressure was less than $100 \mathrm{mmHg}$. Other than pre-medication that may already have been given to patients, no additional betablocker was administered.

For all patients, an iodinated contrast agent (Iopamidol, $370 \mathrm{mgI} / \mathrm{ml}$ Iopamiron; Bayer Healthcare, Osaka, Japan) with a patient weight-based volume $(0.7 \mathrm{ml} \times$ patient weight $(\mathrm{kg}))$ was administered. The contrast agent was injected for $10 \mathrm{~s}$ followed by a $40 \mathrm{ml}$ saline flush. Contrast agent and saline were injected at a rate of 4-5 ml/s into the antecubital vein via a 20 -gauge catheter using a dual-head injector. Automatic bolus tracking was performed with a region of interest (ROI) placed in the aortic root. All CCTA scans were initiated $5 \mathrm{~s}$ after mean ROI contrast reached a pre-set threshold of 200 Hounsfield units (HU).

All patients underwent retrospectively ECG gated helical scans with ECG tube current modulation. The detector collimation was $2 \times 128 \times 0.625 \mathrm{~mm}$, with a dynamic z-focal spot (ZFS), resulting in a sample collimation of 256 simultaneous slices of $0.625 \mathrm{~mm}$ thickness. The tube voltage was 100 or $120 \mathrm{kVp}$ and the gantry rotation time was $0.27 \mathrm{~s}$. An effective tube currentrotation time product (normalized to the pitch factor) ranging between 800 and 1050 mAs (depending on patient habitus) was used for the acquisition. Images were reconstructed with an individually adapted field of view (FOV) encompassing the heart, a matrix size of $512 \times 512$ pixels, an enhanced semi-smooth reconstruction kernel (CB), and a section thickness of $0.67 \mathrm{~mm}$ with an increment of $0.34 \mathrm{~mm}$. The standard temporal resolution of $135 \mathrm{msec}$ was further optimized via advanced adaptive multicycle reconstructions combining data from consecutive cardiac cycles [4]. A dedicated cardiac gating algorithm (Beat-to-Beat Variable Delay Algorithms, Philips Healthcare, Cleveland $\mathrm{OH}$ USA) [5] was used, that enabled the detection and reconstruction of the same physiological cardiac phase of interest. In addition,

Table 1: patient characteristics.

\begin{tabular}{|l|c|}
\hline Number of patients & 20 \\
\hline Age (years) & $66.0 \pm 12.2$ (range 28-81) \\
\hline Body mass index $\left(\mathrm{kg} / \mathrm{m}^{2}\right)$ & $25.3 \pm 4.1($ range 20.8-33.2) \\
\hline Male gender & $14(70 \%)$ \\
\hline Hypertension & $13(65 \%)$ \\
\hline Dyslipidemia & $7(35 \%)$ \\
\hline Diabetes & $13(65 \%)$ \\
\hline Family history of CAD & $4(20 \%)$ \\
\hline Cigarette smoking & $11(55 \%)$ \\
\hline End-stage renal disease & $3(15 \%)$ \\
\hline Peripheral vascular disease & $2(10 \%)$ \\
\hline
\end{tabular}
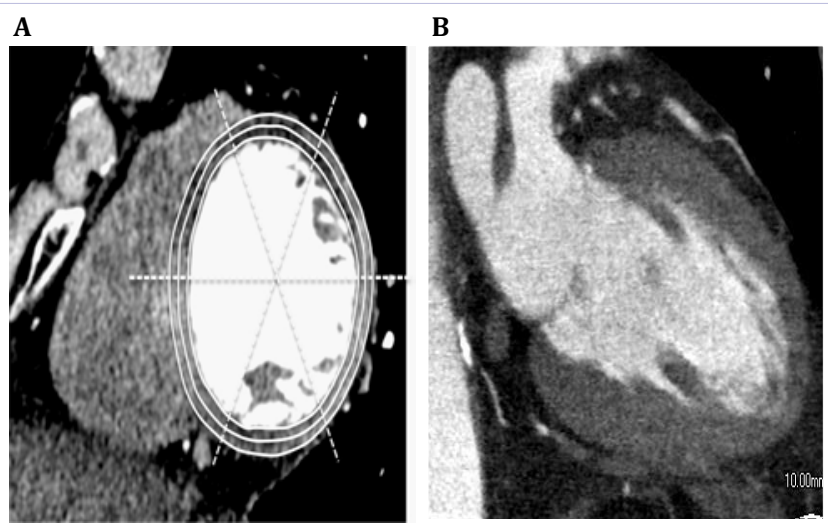

Figure 1: Measurement of attenuation of endocardium and epicardium. The regions of interest (ROIs) in endocardium and epicardium were placed semi-automatically on 2D short-axis (A) and long-axis (B) cardiac images, according to American Heart Association classification. The myocardial CT values for 17 segments were measured at $0 \%, 40 \%, 60 \%$ and $80 \%$ phases.

dynamic helical z-collimation (Eclipse DoseRight Collimator, Philips Healthcare, Cleveland, OH, USA) were used that reduced z-overscanning at the beginning and the end of the helical scans by approximately $25 \%$ for typical cardiac scan lengths [6].

\section{Myocardial Perfusion Imaging (CT-MPI)}

The measurement of myocardial perfusion was performed using the time volume analysis tool on a workstation (TeraRecon Aquarius version 4.4.7, TeraRecon). Image reconstruction of CT-MPI was retrospectively gated to the ECG; 10 phases were reconstructed throughout the cardiac cycle, with the R-R interval divided into $10 \%$ incremental intervals. Myocardial attenuation was assessed using the American Heart Association/American College of Cardiology classification of 17 segment model [7], using LV short-axis and long-axis images with a slice thickness of $2 \mathrm{~mm}$. Myocardial wall thickness for each segment was semiautomatically measured at 4 phases $(0 \%, 40 \%, 60 \%$, and $80 \%$ of the R-R interval). The line in half of the myocardial thickness was manually drawn and separated to subendocardium and subepicardium. Myocardial attenuation (Hounsfield unit; HU) for each segment was measured in the region of interest defined in the endocardium and epicardium at 4 phases $(0 \%, 40 \%, 60 \%$, and $80 \%$ of the R-R interval) Figure 1 . The mean myocardial attenuation (mean MYO) and Standard Deviation (SD) were calculated, for 136 areas in each patient. We defined SSH if the following 2 criteria were met. 1) subendocardial myocardial attenuation at $40 \%$ phase of R-R interval $=<$ mean $\mathrm{MYO}-1 \mathrm{SD}$ and 2) subendocardial myocardial attenuation at $40 \%$ phase of $\mathrm{R}-\mathrm{R}$ interval $=<$ subepicardial myocardial attenuation at $40 \%$ phase of R-R interval. All segments were classified into RCA, LAD and LCX territories.

\section{Invasive Coronary Angiography Acquisition and Quantitative Coronary Analysis}

Invasive Coronary Angiography (ICA) was performed using standard orthogonal views and was clinically driven. Coronary angiographic images were transferred to an independent 
angiographic core laboratory for analysis. The coronary tree was analyzed using a 14-segment coronary model as previously described. A vessel supplying a territory was considered obstructive if at least 1 segment of a vessel contained $a>50 \%$ and $>75 \%$ luminal stenosis.

\section{Statistical analysis}

The diagnostic capability for the detection of CAD was analyzed using the agreement with the territories with SSH and the stenotic coronary territories on CAG.

Diagnostic capability of SSH to detect CAD, based on both $>50 \%$ and $>75 \%$ luminal stenotic territory on CAG, was calculated. SSH was considered to be correct if SSH were seen in the same myocardial segments with coronary stenosis on CAG. We calculated sensitivity, specificity, positive predictive value, and negative predictive value for SSH.

\section{Results}

\section{6-MSCT CCTA}

Of the 60 territories, 28 had SSH on CCTA as defined by our criteria; 8 SSHs to the Right Coronary Artery (RCA), 16 SSHs to the Left Anterior Descending Artery (LAD), and 4 SSHs to the left circumflex branch (LCX). Of the 20 patients, 19 had SSH. There were 9 patients with two-vessel disease and 1 patient with triplevessel disease.

\section{CAG}

Of the 60 territories, 38 had $>50 \%$ stenosis and 30 had $>75 \%$ stenosis on CAG by our criteria; 10 areas with $>50 \%$ stenosis to the Right Coronary Artery (RCA), 18 areas with $>50 \%$ stenosis to the left anterior descending artery (LAD), 10 areas with $>50 \%$ stenosis to the Left Circumflex branch (LCX), 9 areas with $>75 \%$ stenosis to the right coronary artery (RCA), 17 areas with $>75 \%$ stenosis to the left anterior descending artery (LAD), 4 areas with $>75 \%$ stenosis to the left circumflex branch (LCX). Overall, all 20 patients had $>50 \%$ stenosis and 18 of them had $>75 \%$ stenosis.

\section{Diagnostic capability of SSH}

Table 2 shows that $>50 \%$ stenosis-level diagnostic performance data of SSH to detect stenotic coronary territory. Using our criteria, SSH identified 24 of 38 territories with $=>50 \%$ stenosis and 22 of 30 territories with $=>75 \%$ stenosis SSH identified stenotic territory with a modest sensitivity and good specificity. When SSH was compared with either $=>50 \%$ stenosis or $=>75 \%$ stenosis, sensitivity improved but specificity decreased. Accuracy of $=>75 \%$ stenosis detection was higher than accuracy of $=>50 \%$ stenosis detection Table 3 .

In our study, the discrepancy of coronary stenosis between CCTA versus CAG was observed in 12 coronary territories; 2 territories of over diagnosis, 1 territory of under diagnosis, 9 territories of not assessed due to severe calcified plaques. The use of the SSH sign led to accurate diagnosis (result of CAG) in 10 of these 12 territories. Representative cases were indicated in Figures 2 and 3. Nine territories of 60 territories (15\%) could not be assessed with CCTA, whereas assessed territories could be assessed with both CCTA and SSH.
Table 2: The incidence of SSH and the stenotic coronary territories on CAG.

\begin{tabular}{|l|c|c|c|}
\multirow{2}{*}{ Total } & CCTA & \multicolumn{2}{|c|}{ CAG } \\
\cline { 2 - 4 } & SSH(+) & $>50 \%$ & $>75 \%$ \\
\hline Patient & $19 / 20(95 \%)$ & $20 / 20(100 \%)$ & $18 / 20(90 \%)$ \\
\hline \multirow{2}{*}{ Vessel territory } & $28 / 60(47 \%)$ & $38 / 60(63 \%)$ & $30 / 60(50 \%)$ \\
\hline RCA & CCTA & CAG & \\
\cline { 2 - 4 } & SSH $(+)$ & $>50 \%$ & $>75 \%$ \\
\hline LAD & $8 / 20(40 \%)$ & $10 / 20(50 \%)$ & $9 / 20(45 \%)$ \\
\hline & $16 / 20(80 \%)$ & $18 / 20(90 \%)$ & $17 / 20(85 \%)$ \\
\hline
\end{tabular}

Table 3: CAD diagnostic capability of SSH by coronary territory base.

\begin{tabular}{|l|l|l|}
\hline \multirow{2}{*}{} & \multicolumn{2}{|l|}{ The threshold value of a Coronary Stenosis (CAG) } \\
\cline { 2 - 3 } & $\mathbf{5 0 \%}$ & $\mathbf{7 5 \%}$ \\
\hline Sensitivity & $63.2 \%(24 / 38)$ & $73.3 \%(22 / 30)$ \\
\hline Specificity & $81.8 \%(18 / 22)$ & $80 \%(24 / 30)$ \\
\hline PPV & $85.7 \%(24 / 28)$ & $78.6 \%(22 / 28)$ \\
\hline NPV & $56.3 \%(18 / 32)$ & $75 \%(24 / 32)$ \\
\hline Accuracy & $70 \%(42 / 60)$ & $76.7 \%(46 / 60)$ \\
\hline
\end{tabular}
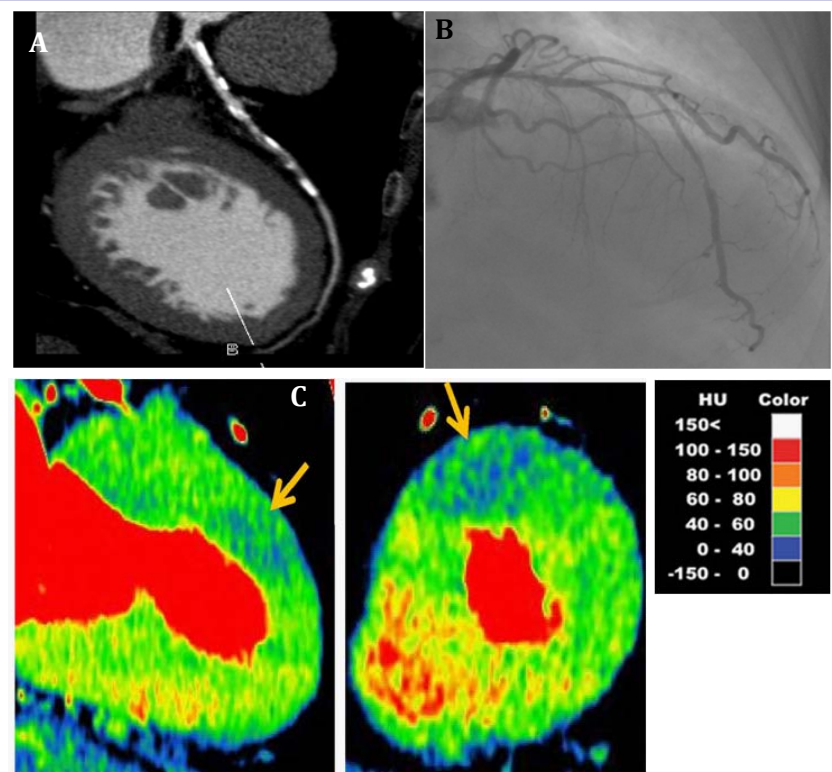

Figure 2: Severe calcified plaque and significant stenosis at LAD territory in a female patient aged 81 years.

A: Coronary computed tomography angiography (CCTA) showed severe calcified plaque at LAD territory; but degree of luminal stenosis is unclear.

B: CAG showed stenotic lesion at segment 7 (LAD territory)

C: CT perfusion images of systolic phase showed subendocardial systolic hypoperfusion at anterior wall (LAD territory). CT value of endocardium was $60 \mathrm{HU}$. CT value of epicardium was $62 \mathrm{HU}$. Mean MYO was $89 \mathrm{HU}$ (SD is $8 \mathrm{HU}$ ), thus cut-off was $81 \mathrm{HU}$. SSH sign was positive. SSH positive territory was identical with coronary stenotic territory.

\section{Discussion}

Our study demonstrated the potential of 256-slice MSCT incorporating coronary angiogram and perfusion imaging enables consistent detect of coronary stenotic territory, as verified via CAG. Identification of coronary stenosis is important 


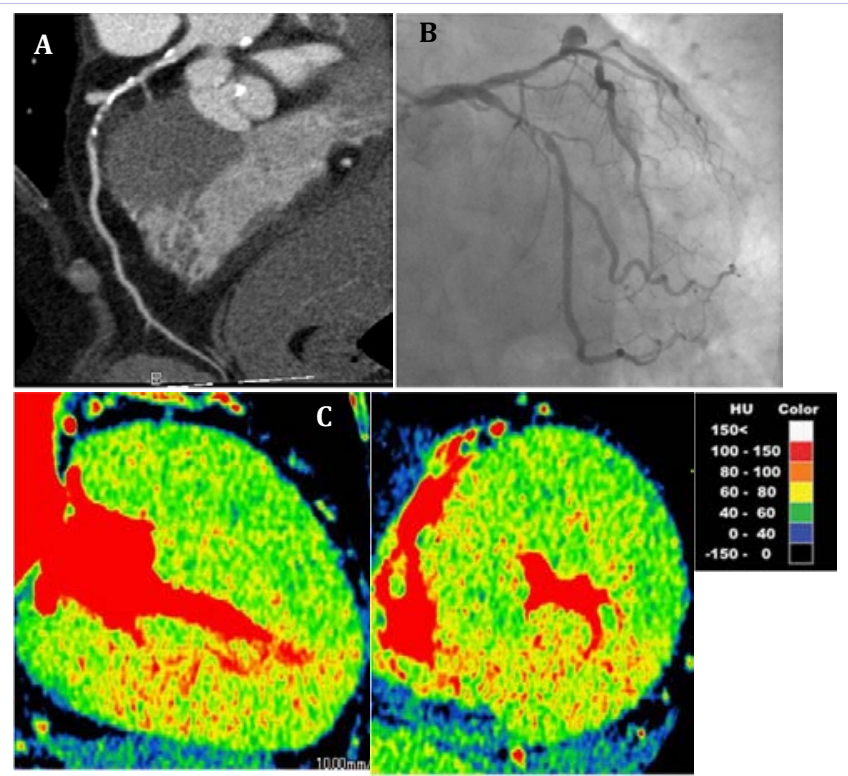

Figure 3: Severe calcified plaque in the LAD, but no stenosis in a female patient aged 79 years

A: Coronary Computed Tomography Angiography (CCTA) showed severe calcified plaque at LAD territory; but degree of luminal stenosis is unclear B: CAG showed no stenotic lesion at LAD territory.

C: CT perfusion images of systolic phase showed no subendocardial systolic hypoperfusion. CT value of endocardium was $85 \mathrm{HU}$. CT value of epicardium was $81 \mathrm{HU}$. Mean MYO was $87 \mathrm{HU}$ (SD is $7 \mathrm{HU}$.), thus cut-off was $80 \mathrm{HU}$. SSH sign was negative. SSH negative territory was indicative of absence of significant CAD.

to determine prognosis and to plan patient treatments. Using resting CTA images, myocardial perfusion evaluation is possible without the need for further studies, or additional radiation or contrast. This study showed the novel quantitative method to detect coronary stenotic territory with resting CTA images.

The use of the SSH sign led to accurate diagnosis (result of CAG) in 10 of the 12 territories deviated coronary stenosis between CCTA versus CAG. The presence of SSH might provide useful additional information when the assessment of coronary artery is made difficult by severe calcification.

The decline of myocardial contrast enhancement is due to reduction of myocardial blood flow in the coronary artery supplying blood to the ischemic region of the myocardium. The endocardium is the area of the left ventricle most vulnerable to the effects of hypoperfusion and ischemia. As the result, the ischemic area exhibits subendocardial low attenuation (myocardial enhancement) compared to the normal regions [1].

Although several previous studies have demonstrated possibility of rest CTP imaging to identify myocardial ischemia Spiro AJ et al $[3,8]$. stated that resting cardiac 64-MDCT dose not reliably detect myocardial ischemia identified by radionuclide imaging [9]. The mean body mass index for their patients was 30 , supposing that the majority of patients were obese. This affects increase in signal noise and decrease in myocardial enhancement, resulting miss - diagnosis by rest CTP imaging. On the other hand, the mean body weight for our Japanese patients was about $60 \mathrm{~kg}$, and the mean body mass index was about 20 . Patient size is suitable for getting high contrast and artifact less image. In addition, the scan time of 256-slice MSCT is less than 5 seconds and that of 64-slice, and the timing is the early phase of myocardial enhancement during the first pass. The short scan time of 256-slice MSCT can amplify the difference in myocardial enhancement between systole and diastole. Further, 256-slice CT with high temporal resolution can achieve motion artifact less in myocardial imaging at systole as well as diastole.

With a standard temporal resolution of $135 \mathrm{~ms}$ further optimized via cardiac adaptive multi-cycle reconstructions, the end-systolic rest phase can be successfully targeted using the 256-slice MSCT enabling the user to accurately trace myocardial boundary. With a wide coverage of $8 \mathrm{~cm}$, the contrast bands are minimized, thus also reducing their impact on attenuation changes $[10,11]$. The resulting short scan times of 256-slice MSCT ( $<5 \mathrm{~s}$ for a typical cardiac anatomy) could facilitate optimization of the contrast volume needed to help enable cardiac imaging at the peak coronary enhancement [12].

Limitations: This was single-center pilot study with a small number of patients. The CCTA scans were triggered based on a pre-determined threshold of the mean $\mathrm{HU}$ of the contrast arrival in the aortic root - while this is targeting the early phase of the myocardial enhancement during the first pass, the timing may not be optimized for the peak enhancement of the myocardium. In addition, our investigations did not quantify the measures commonly associated with perfusion (blood flow, volume, etc). These are typically extracted from dynamic perfusion scans. However, since these scans could result in an increased radiation exposure, we limited our investigations to a single CCTA scan in the interest of keeping the radiation exposure low.

In conclusion, SSH was quantified by subendocardial and subepicardial attenuation using retrospective resting ECGgate CCTA. SSH was present in a high rate in stenotic coronary territories confirmed by $\mathrm{CAG}$, and could provide additional hemodynamic information with the detection of CAD (especially $=>75 \%$ stenosis) with high diagnostic accuracy.

\section{Acknowledgement}

"This work was supported by the Japan Society for the Promotion of Science (JSPS) KAKENHI (25461831)".

\section{References}

1. Higuchi K, Nagao M, Matsuo Y, et al. Evaluation of chronic ischemic heart disease with myocardial perfusion and regional contraction analysis by contrast-enhanced 256-MSCT. Japanese journal of radiology. 2013; 31(2): 123-132.

2. Nagao M, Matsuoka $H$, Kawakami $H$, et al. Myocardial ischemia in acute coronary syndrome: assessment using 64-MDCT. AJR American journal of roentgenology. 2009; 193(4): 1097-1106.

3. Nagao M, Matsuoka H, Kawakami H, et al. Quantification of myocardial perfusion by contrast-enhanced 64-MDCT: characterization of ischemic myocardium. AJR American journal of roentgenology. 2008; 191(1): 19-25. 
4. Manzke R, Grass M, Nielsen T, Shechter G, Hawkes D. Adaptive temporal resolution optimization in helical cardiac cone beam CT reconstruction. Medical physics. 2003; 30(12): 3072-3080.

5. Vembar M, Garcia MJ, Heuscher DJ, et al. A dynamic approach to identifying desired physiological phases for cardiac imaging using multislice spiral CT. Medical physics. 2003; 30(7): 1683-1693.

6. Walker MJ, Olszewski ME, Desai MY, Halliburton SS, Flamm SD. New radiation dose saving technologies for 256-slice cardiac computed tomography angiography. Int J Cardiovas Imag. 2009; 25: 189-199.

7. Cerqueira MD, Weissman NJ, Dilsizian V et al. Standardized myocardial segmentation and nomenclature for tomographic imaging of the heart:A statement for healthcare professionals from the Cardiac Imaging Committee of the Council on Clinical Cardiology of the American Heart Association. Circulation. 2002; 105(4): 539-542.

8. Busch JL, Alessio AM, Caldwell JH et al. Myocardial hypo-enhancement on resting computed tomography angiography images accurately identifies myocardial hypoperfusion. Journal of cardiovascular computed tomography. 2011; 5(6): 412-420.

9. Spiro AJ, Haramati LB, Jain VR, Godelman A, Travin MI, Levsky JM. Resting Cardiac 64-MDCT Does Not Reliably Detect Myocardial Ischemia Identified by Radionuclide Imaging. Am J Roentgenol. 2013; 200(2): 337-342.

10.Herzog C, Arning-Erb M, Zangos S, et al. Multi-detector row CT coronary angiography: influence of reconstruction technique and heart rate on image quality. Radiology. 2006; 238(1): 75-86

11. Klass O, Walker M, Siebach A, et al. Prospectively gated axial CT coronary angiography: comparison of image quality and effective radiation dose between 64-and 256-slice CT. European radiology. 2010; 20(5): 1124-1131.

12. Bae KT. Intravenous Contrast Medium Administration and Scan Timing at CT: Considerations and Approaches. Radiology. 2010; 256(1): 32-61. 